\title{
Characterization and Relative Ionization Efficiencies of End-Functionalized Polystyrenes by Matrix-Assisted Laser Desorption/Ionization Mass Spectrometry
}

\author{
Frederick J. Cox and Murray V. Johnston \\ Department of Chemistry and Biochemistry, University of Delaware, Newark, Delaware, USA
}

\author{
Arnab Dasgupta* \\ Department of Materials Science and Engineering, University of Delaware, Newark, Delaware, USA
}

\begin{abstract}
The properties and relative ionization efficiencies of a series of polystyrenes (PS) with hydroxyl, hydrogen, tertiary amine, and quaternary amine end-functionalities were examined by matrix-assisted laser desorption/ionization (MALDI) time-of-flight (TOF) mass spectrometry (MS). The hydrogen and hydroxyl functionalized PS ionized via attachment of a single $\mathrm{Ag}^{+}$cation, as expected. However, tertiary amine PS oligomers were found in $(\mathrm{M}-\mathrm{H})^{+}$and $(\mathrm{M}+\mathrm{H})^{+}$forms, in addition to $\mathrm{M}+\mathrm{Ag}^{+}$, while the quaternary amine PS oligomers only ionized to the $\mathrm{M}^{+}$form. Analysis of tertiary amine PS spectra revealed varying ratios of the three ionic forms depending on the oligomer length, pointing to a change in ionization efficiency. When the bulk samples were compared, the quaternary amine end-functionalized PS gave a ten-fold higher ionization efficiency over all others studied, likely because of the preexisting charge on the functionality. Samples with hydroxyl and hydrogen functionalities had similar ionization efficiencies, with the tertiary amine slightly higher, depending on the molecular weight. Changes in molecular weight affected the relative ionization efficiencies in varying fashion depending on the end functionality, though average molecular weight measurements were largely unaffected by end-functionality. Quantification of end-functionalized polystyrenes with different ionization efficiencies was found to be possible if due care was taken. (J Am Soc Mass Spectrom 2003, 14, 648-657) (C 2003 American Society for Mass Spectrometry
\end{abstract}

$\mathrm{M}$ atrix assisted laser desorption/ionization (MALDI) mass spectrometry (MS) has become a well-established method for the analysis of diverse polymeric materials. With proper matrix and cation selection, spectra can easily be obtained containing molecular ions, with or without cation adducts, of a wide range of common polymers such as poly(ethylene oxide) or polystyrene (PS) [1-3]. From the MALDI spectrum, one can usually determine the average molecular weight of the bulk sample and specific molecular weights of oligomers. Polymer repeat units and end groups can then be deduced from the oligomer masses. Because of this rich information provided, MALDI has become preeminent among the means of spectroscopic polymer analysis. Although MALDI analysis of some polymers is often routine, many others present great

Published online May 13, 2003

Address reprint requests to Dr. M. V. Johnston, Department of Chemistry and Biochemistry, University of Delaware, 107 Brown Lab, Newark, DE 19716, USA. E-mail: mvj@udel.edu

${ }^{*}$ Current address: Electronic Chemicals Division, Indium Corporation of America, Clinton, NY 13323. difficulty, as there may be insufficient signal for proper spectral analysis. Even among the few classes of polymers that have been studied in depth, there is still great uncertainty in the mechanisms that permit facile and quantitative MALDI analysis.

Polystyrenes are one such class of polymers in which the MALDI analysis has been explored in depth. Nearly all aspects of the analysis of PS, from ionization to data analysis, have been examined. For example, various studies have explored the effect of sample preparation on PS ionization, including clustering of the metal cation in vacuo [4], oligomer clustering on the sample surface [5], and solid mixed/applied samples [6]. Cation attachment has been explored in numerous reports, with silver generally found to be the most facile cation for ionization of polystyrene oligomers [7, 8]. Others have examined the structure of cationized PS oligomers [9]. Secondary series and adduct formation have been probed using post-source decay in time-of-flight MS [10], and fragmentation of PS oligomers, along with end group identification, has been examined via collision induced dissociation in tandem MS experiments [11- 
13]. Finally, biases in average molecular weights of PS have been studied with regard to increasing discrete oligomer size [14], and in comparison with classical measurements [15-17].

Despite the extensive research on the MALDI of PS, little work has been done on the effect of the end groups on the ionization. Generally, it has been assumed that the end groups have little effect on the ionization efficiency of PS, such as when MALDI has been used to quantify products with differing end-functionality following the polymerization of styrene. This assumption was used by both Quirk et al., who quantified the ethylene oxide oligomerization during end-functionalization of poly(styryl)lithium showing good agreement with data obtained by ${ }^{1} \mathrm{H}$ NMR [18], and by Zammit et al., who quantified the mode of termination of a free radical polymerization of styrene and found good agreement with multiple previous studies using other methods [19]. The assumption was valid in both cases, i.e., quantification of oligomers with different end groups by MALDI showed no obvious bias towards a particular end group, and as such no detailed investigation of an end group bias was done. In one case where the influence of end functional groups was quantified, Belu and coworkers explored the relative ionization yields of dimethylphenylsilyl- and perfluoroalkylsilyl- terminated PS as compared to that terminated with a proton but found little difference in the aggregate values [20]. In another study, Weaver and coworkers explored the quantification of hydroxyl- and hydrogen-terminated polystyrene, finding equal yields of the polymers for equal molar amounts. However, they determined MALDI to be unsuitable for quantification due to biases toward the hydroxyl end group when the molar amount of the hydroxyl-terminated polymer was much greater than that of the hydrogenterminated polymer [21].

Recent research with other polymers, however, suggests that end groups may play an important role in ionization and can change the efficiency significantly. For example, the ionization efficiencies of Nylon 6 and polybutyleneterephthalate oligomers have been found to vary greatly depending on the end group [22, 23]. Perhaps more significantly, the addition of a so-called "covalent cation" - an organic cation, such as triphenylphosphine, covalently bonded to an oligomer as an end group - permits the ionization of polyolefins which previously gave little or no signal in normal MALDI analysis [24, 25]. Similarly, polyisobutylene that has been end-sulfonated ("covalent anion") has been shown to greatly increase ion yield versus unreacted polymer [26]. Thus, the assumption of equivalent ionization efficiencies regardless of the PS end group may not be valid in all cases, and is likely invalid in the case of the covalent cation/anion. If MALDI is to be used for quantification of polymer mixtures, as recent work suggests is practical [27], quantification of any differences in ionization efficiencies between oligomers with different end-functionalities is critical.
Table 1. Samples used in analyses

\begin{tabular}{|c|c|c|c|}
\hline $\begin{array}{l}\text { Sample } \\
\text { Name }\end{array}$ & $\mathrm{Mn}^{\mathrm{a}}$ & End Functionality & Source \\
\hline PSA1600 & 1665 & Tertiary amine ${ }^{b}$ & - \\
\hline PSQ1600 & 1676 & Quaternary amine ${ }^{c}$ & - \\
\hline PSH1600 & 1790 & Hydrogen & Fluka \\
\hline PSOH1600 & 1600 & Hydroxyl $^{d}$ & Polymer Source \\
\hline PSA2500 & 2520 & Tertiary amine $e^{b}$ & - \\
\hline PSQ2500 & 2595 & Quaternary amine ${ }^{c}$ & - \\
\hline PSH2500 & 2500 & Hydrogen & Amer. Poly. Standard \\
\hline PSA14200 & 14253 & Tertiary amine $e^{b}$ & - \\
\hline PSO14200 & 14405 & Quaternary amine ${ }^{c}$ & - \\
\hline
\end{tabular}

aNumber average molecular weight are as reported by manufacturer for commercial samples, and obtained by MALDI-MS for all others.

b- $\left(\mathrm{CH}_{2}\right)_{3} \mathrm{~N}\left(\mathrm{CH}_{3}\right)_{2}$.

c $-\left(\mathrm{CH}_{2}\right)_{3} \mathrm{~N}\left(\mathrm{CH}_{3}\right)_{3}{ }^{+}$.

d- $\mathrm{C}_{2} \mathrm{H}_{4} \mathrm{OH}$.

Here we explore a series of end-functionalized polystyrenes that do exhibit significant differences in ionization efficiency. The MALDI spectra of end-functionalized polystyrenes (hydroxyl, hydrogen, and tertiary/ quaternary amine) of similar molecular weight and polydispersity are compared and the relative ionization efficiencies quantified. Because a single tertiary amine end-functionalized PS oligomer can be ionized in multiple modes (silver cationized, protonated, and covalently) depending on sample preparation, it provides a convenient case for investigation of the effect of the end group. The effect of laser power on the relative ionization efficiency of the various polystyrenes is also investigated. In order to determine whether the end groups have any effect on average molecular weight values, the molecular weights of the tertiary amine end-functionalized PS are compared. Finally, the implications of the differences in ionization efficiencies on polymer analysis are discussed.

\section{Experimental}

\section{Materials}

Details of the synthesis of the amine end functionalized polystyrenes will be described in a separate communication [28]. Briefly, tertiary amine polystyrenes (PSA) of varying molecular weight $(\mathrm{Mn}=1600$ to 14,000$)$ were synthesized via anionic polymerization of styrene with sec-butyl lithium initiator followed by addition of the propyl amine chloride in the presence of $\mathrm{LiCl}$ [29]. Quaternization of the dimethyl amine was achieved through subsequent reaction with methyl iodide or dimethyl sulfate to add a third methyl group, with the dimethyl sulfate being the preferred method giving quantitative results. Quantitative $(100 \%)$ conversion of the amine to quaternary form (PSQ) was confirmed by thin-layer chromatography. Average molecular weights were determined on the tertiary and quaternary amines by MALDI (Table 1 ).

Hydroxy (PSOH) and hydrogen (PSH) terminated polystyrenes were purchased from commercial sources 
as indicated in Table 1. Matrix (dithranol) and cationization salt (AgTFA) were purchased from Aldrich (Milwaukee, WI) and used without further purification.

\section{Methods}

All mass spectra were obtained on a Biflex III MALDI time-of-flight mass spectrometer (Bruker, Billerica, MA) in both reflectron $(<5 \mathrm{ku})$ and linear modes using delayed extraction. Matrix ion deflection and detector gating were used to minimize the effects of matrix signal saturation. Spectra were generally an average of 300 discrete laser shots minimum, with sample position moved every 5-10 shots, or after marginal signal loss due to sample depletion. This reduced the effects of any localized signal differences in the spot area. Mass calibration was performed with previously characterized poly para methylstyrene [30] with dithranol and AgTFA in reflectron mode, and a pre-prepared protein mixture (Applied Biosystems, Foster City, CA) and bovine serum albumin with CHCA (Aldrich) in linear mode. All solutions were prepared in toluene at $4 \mathrm{mg} / \mathrm{mL}$. Dithranol was used as a matrix, and AgTFA, when needed, as the cationization salt for the polymer samples. Analyte solutions were premixed at an 8:4:1 volume ratio of matrix to sample(s) to salt, applied in $1 \mu \mathrm{L}$ aliquots to the probe, and allowed to air dry. Because high quality spectra were achieved by this technique, alternate sample preparation methods were not explored.

For determining relative ionization efficiencies, multiple standard solutions of hydrogen, hydroxyl, tertiary amine, and quanternary amine functionalized PS were prepared at $4 \mathrm{mg} / \mathrm{mL}$ and mixed with each other in varied ratios. Specific components in each mixture were chosen so that no isobaric interferences would be present in the resultant spectra. Where interference was present, mixtures were made so that comparison of relative efficiency could be made using separate mixtures of each component with a third distinct component. The mixtures were then mixed with dithranol (and AgTFA when indicated), spotted on the MALDI plate, and spectra recorded. To minimize any possible effects of changing the ratio of matrix to analyte to cation, all mixtures were made up so that the relative total amount of analyte in mixtures was the same as would be used in a single component analysis. Spectra at a particular ratio were usually obtained nine times to ensure that any one sample was representative of the "actual" value: Three positions on the plate were spotted from each mixture, and for each ratio, three mixtures were made. Each of the three mixtures was from separately prepared solutions of the analytes (although same matrix and salt solutions) in order to eliminate propagation of any determinate error from the preparation of a single standard solution.

Deisotoping, molecular weight calculations, and oligomer ion intensity integrations were performed using the Polymerix software package (Sierra Analytics, Modesto, CA). Relative ionization efficiencies, regres- sions, and error analyses were calculated using Excel (Microsoft, Redmond, WA) on data exported from Polymerix.

\section{Results and Discussion}

\section{End Functionalized Polystyrenes}

The MALDI-MS spectra of both hydrogen and hydroxyl terminated polystyrene have been explored extensively in other studies, and are similar to each other [10, 18, 21, 31]. Both easily ionize by attachment of a silver cation, giving a single isotopic distribution for each oligomer, separated by $104.1 \mathrm{~m} / \mathrm{z}$. The PSH and PSOH samples we examined here differed little from those published previously, giving reproducible spectra of high signalto-noise when mixed with dithranol and AgTFA. Although other researchers have reported significant byproducts of the hydroxyl termination reaction in MALDI-TOF MS analysis of similarly terminated polystyrenes [18, 21], no such byproducts were evident in our experiments with PSOH. No modes of ionization other than silver cationization were detected, but small intensity secondary series (as from adduct formation and decay) were observed in agreement with previous studies of butyl terminated PS [10].

Amine functionalized polystyrenes have been explored to a lesser extent. Jackson et al. examined the MALDI-MS spectra of polystyrene with a tertiary amine end group using all-trans-retinoic acid as a matrix, observing protonated species and, when a copper salt was added to the analyte, copper ion adducts [13]. As expected, using dithranol as a matrix, PSA was found to be easily ionizable, both without and with AgTFA, as shown in Figures 1 and 2, respectively. Each set of peaks is the overlap of isotope distributions from multiple species arising from a single oligomer length. Unlike the previous study, however, the isotopic distribution obtained here indicated the possible presence of a deprotonated species and/or a radical cation, in addition to the protonated molecule and metal adduct. In order to positively identify species and determine their relative amounts in both spectra, theoretical distributions of the possible expected isotopes, $\mathrm{M}-\mathrm{H}^{+}$, $\mathrm{M}^{+}, \mathrm{M}+\mathrm{H}^{+}$, and $\mathrm{M}_{\mathrm{n}-1}+\mathrm{Ag}^{+}$, were compared to the experimental isotope distributions. Additionally, the distributions were deisotoped, and the resulting monoisotopic peaks identified. Without silver, only $\mathrm{M}-$ $\mathrm{H}^{+}$and $\mathrm{M}+\mathrm{H}^{+}$were observed (Figure 1). Upon addition of AgTFA, an additional series of $\mathrm{M}_{\mathrm{n}-1}+\mathrm{Ag}^{+}$ ions was also observed (Figure 2). No $\mathrm{M}^{+\cdot}$ ions were determined to be present in any appreciable quantity after deisotoping, both with and without AgTFA. The $\mathrm{M}+\mathrm{H}^{+}$ion is likely a simple protonation at the amine, while the $\mathrm{M}-\mathrm{H}^{+}$likely results from the loss of $\mathrm{H}_{2}$ by formation of the imine from the $\mathrm{M}+\mathrm{H}^{+}$ion. A number of secondary ion series are also observed. Well resolved, though not intense, peaks in samples without silver were observed at both $\mathrm{M}+17 \mathrm{~m} / \mathrm{z}$ and $\mathrm{M}-13 \mathrm{~m} / \mathrm{z}$ (or 


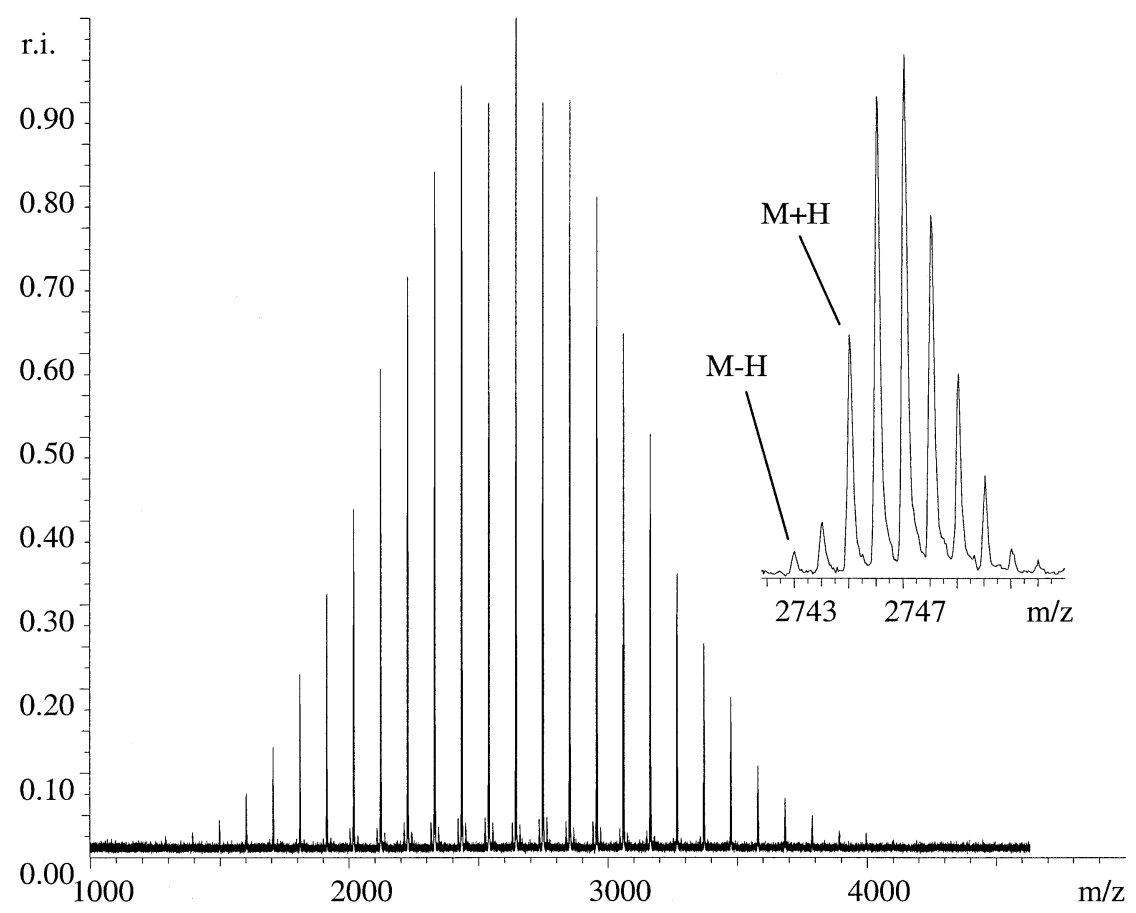

Figure 1. MALDI-MS spectrum of PSA2500, without silver. Inset is isotopic distribution of 25 mer oligomer.

$\mathrm{M}+91 \mathrm{~m} / \mathrm{z}$ ) modulo $104 \mathrm{u}$, whose identities have not been determined. Interestingly, preliminary MALDIFT-MS spectra of the amine functionalized samples have similar features, although $\mathrm{M}-\mathrm{H}^{+}$ions are present in much higher quantity, with concurrent decrease in $\mathrm{M}+\mathrm{H}^{+}$signal, likely because of the longer analysis time (relative to TOF-MS) permitting decay to the $\mathrm{M}-\mathrm{H}^{+}$form (unpublished data).

A partially resolved secondary ion series was also observed $14 \mathrm{~m} / \mathrm{z}$ higher than $\mathrm{M}^{+\cdot}$ (or $90 \mathrm{~m} / \mathrm{z}$ below), but only in tertiary amine spectra containing AgTFA. However, the isotopic distribution does not support inclu-

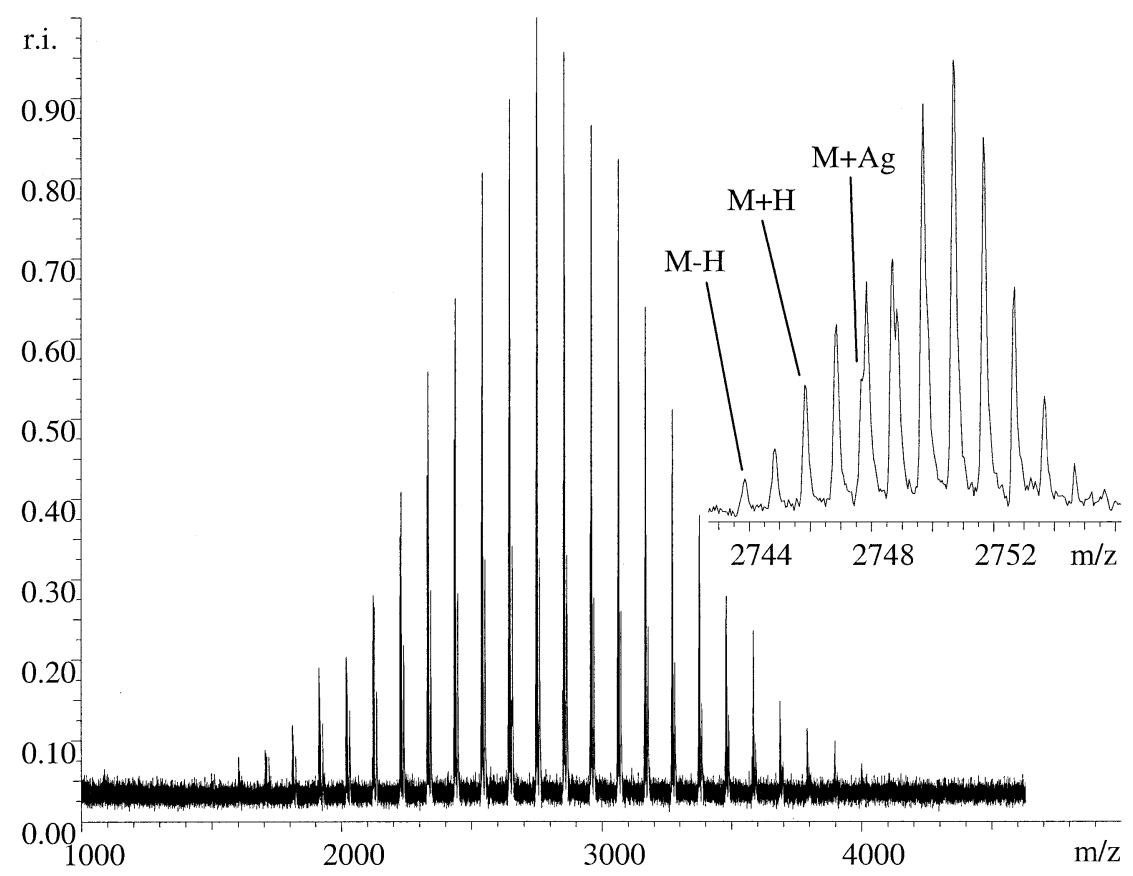

Figure 2. MALDI-MS spectrum of PSA2500, with AgTFA added. Note additional peaks from silver cationization in the inset absent in Figure 1. 


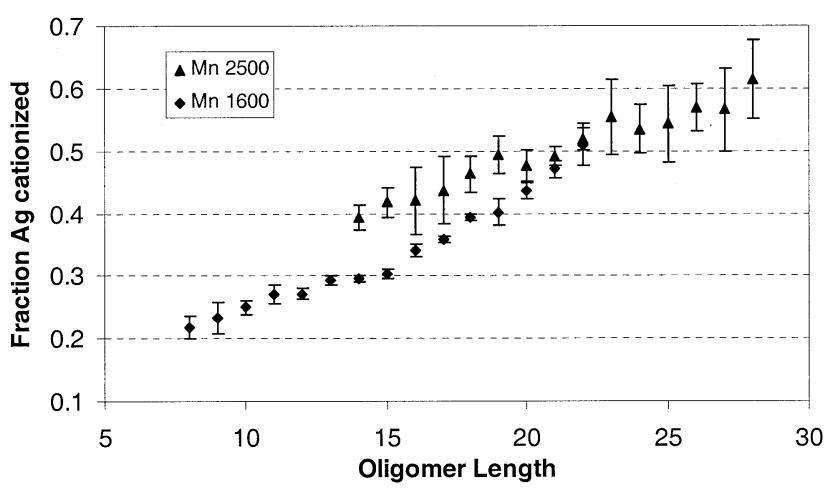

Figure 3. Fraction of $\mathrm{Ag}^{+}$oligomers as a function of oligomer length, as determined from PSA1600 and 2500. Error bars represent one standard deviation at each point.

sion of silver in the ion. The intensity of this series increased relative to the main series with increasing laser power, and average molecular weight values were generally equivalent to that of the major series. These results and the absence of this series in the linear mode suggest that it is likely a metastable decomposition product. As the ionization potentials of the silver atom and a tertiary amine are comparable, $7.58 \mathrm{eV}$ for silver and $7.70 \mathrm{eV}$ for $\mathrm{N}, \mathrm{N}$-dimethyl-benzeneethanamine [32], it is possible that charge exchange occurs, leading to an $\mathrm{M}^{+}$'ion with further reaction to the observed ion series. However, the identity of the ion series has not been determined, and does not seem to occur through any easily described mechanism.

The relative ratios of $\mathrm{M}-\mathrm{H}^{+}, \mathrm{M}+\mathrm{H}^{+}$, and $\mathrm{M}+$ $\mathrm{Ag}^{+}$peaks in the isotopic distributions of any particular oligomer in a spectrum of PSA with AgTFA were found to vary depending on size of the oligomer. In order to quantify this phenomenon, multiple spectra for two samples, PSA1600 and PSA2500, both with AgTFA, were obtained. Spectra were centroided and deisotoped, and then the values of the various ionic forms for a given oligomer were totaled and the silver cationization calculated as a mole fraction and averaged (Figure 3 ). Over the range of oligomer lengths 8 to 28 , the mole fraction that is silver cationized increases with increasing oligomer length in a linear fashion $\left(\mathrm{R}^{2}=0.96\right.$ for each data set), and is found to be very reproducible. Although the data for both the PSA1600 and PSA2500 do not precisely overlap, the slopes are roughly parallel, and the absolute values at multiple oligomer lengths are similar. Values for higher molecular weights could not be obtained because of the error in the deisotoping procedure resulting from insufficient resolution of peaks.

The most likely explanation for this trend is oligomer length-dependent efficiency of silver cationization. Kinugasa and coworkers have observed a trend of increasing ionization efficiency with increasing oligomer size for equimolar amounts of monodisperse polystyrene oligomer with silver cation, albeit ending at lower oligomer lengths than studied here [14]. Cox and coworkers saw a similar effect with skewed composition values for poly(isobutylene-co-para-methylstyrene) copolymers, as oligomers with increasing numbers of methylstyrene monomer appeared to be preferentially cationized [30]. Conversely, the efficiency of protonation may decrease with increasing oligomer length. However, as the number of sites for protonation is the same regardless of oligomer length, while the number of sites for cation attachment increase with each additional oligomer, the first case seems more likely. In either case, one would expect changes in ionization efficiency dependent on oligomer length to be reflected in the average molecular weights of the various ionic forms. If silver cationization increases with oligomer length, the average molecular weight of the silver cationized oligomers would be skewed relative to that of the protonated oligomers.

Table 2 shows the number average molecular weights of the tertiary samples with and without AgTFA, and sample with AgTFA divided into the component molecular weights arising from the protonated and silver cationized forms of the oligomers. When the average molecular weights arising from both molecular forms are isolated by deisotoping from the composite spectrum, the number average weight of the silver cationized oligomers is significantly higher than the composite weight, and the weight of the protonated oligomers slightly lower. If the skewing resulted from a competitive ionization process, and the silver cationization occurred solely instead of protonation, one would expect the average molecular weight of the protonated oligomers to decrease when AgTFA was added, as fewer higher molecular weight oligomers would be protonated. However, there is no significant difference in the average molecular weight of the protonated oligomers with or without AgTFA, indicating that protonation is unaffected by silver cationization. Thus, the increase in silver cationization at longer oligomer lengths does not occur at the expense of the protonated

Table 2. Number average molecular weights ${ }^{\mathrm{a}}$ of different ionization forms of amine functionalized PS

\begin{tabular}{|c|c|c|c|c|}
\hline Sample name & $\begin{array}{c}\text { Composite Mn } \\
\text { w/ AgTFA }\end{array}$ & $\mathrm{Mn}(\mathrm{M}+\mathrm{Ag})^{+} \mathrm{w} / \mathrm{AgTFA}$ & $\begin{array}{c}\mathrm{Mn}(\mathrm{M}+\mathrm{H})^{+} \\
\text {and }(\mathrm{M}-\mathrm{H})^{+} \\
\text {w/ AgTFA }\end{array}$ & Composite $\mathrm{Mn}$ w/o AgTFA \\
\hline PSA1600 & $1663 \pm 3$ & $1720 \pm 4$ & $1636 \pm 4$ & $1647 \pm 4$ \\
\hline PSA2500 & $2518 \pm 11$ & $2562 \pm 28$ & $2469 \pm 21$ & $2460 \pm 30$ \\
\hline
\end{tabular}

${ }^{a}$ Molecular weights were calculated after centroiding and deisotoping. Contributions from the mass of the silver in adducts have been subtracted. 


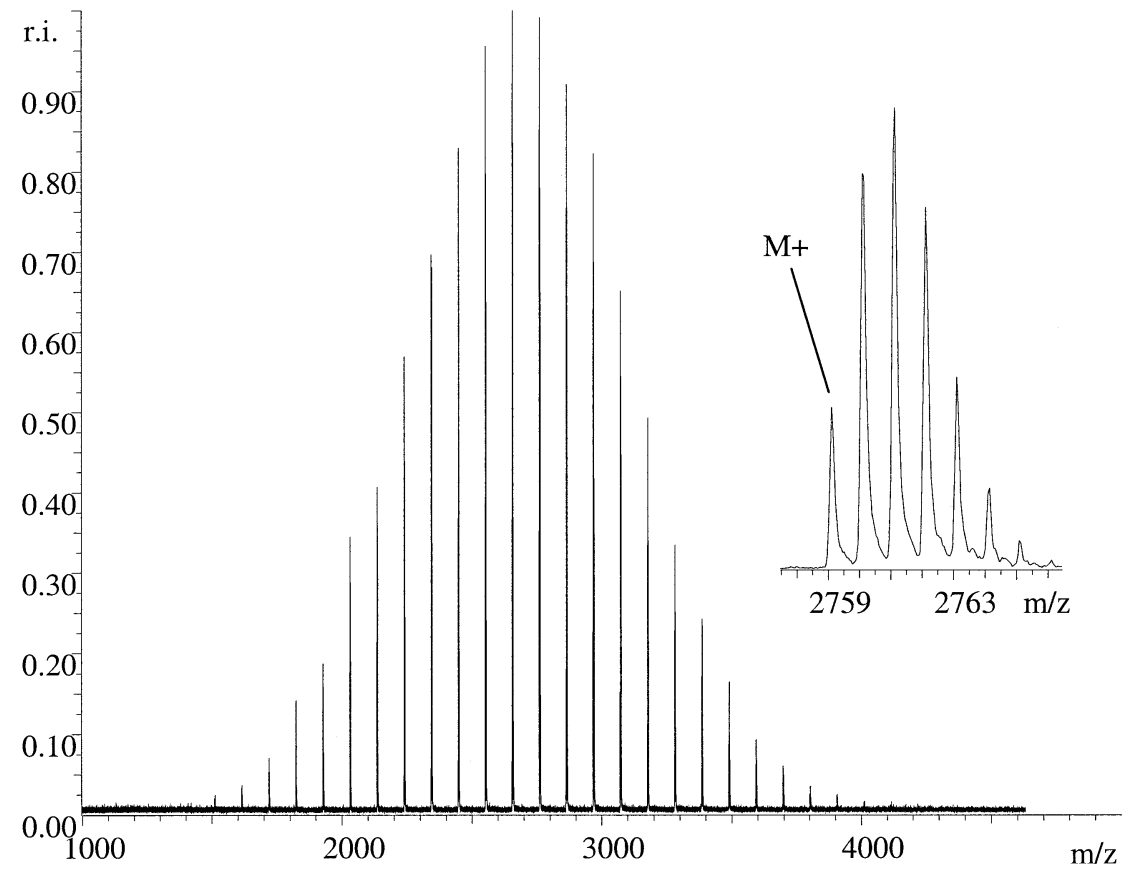

Figure 4. Spectrum of PSQ2500. Signal to noise ratio is significantly higher than in Figures 1 or 2.

form, but in the ionization of additional oligomers, pointing to an increase in total ion yield.

Spectra of quaternary amine polystyrenes, shown in Figure 4, are easily obtained with dithranol matrix, and give only a single intense peak $\left(\mathrm{M}^{+}\right)$for each oligomer. The addition of AgTFA resulted in no visible change in the spectrum, other than the requisite silver cluster formation, which was appreciably higher than normally encountered with PS samples at similar concentration. This likely occurs because there are no silver cations bound to PS oligomers, thus leading to higher concentrations of free $\mathrm{Ag}^{+}$and increased clustering. No secondary ion series were observed. As compared to the tertiary amine, lower laser power was required for the quaternary amine samples to realize similar signal-tonoise ratios in the spectra. Significant increases in signal were also observed over those obtained from the tertiary amine at equivalent laser powers and concentrations, beyond that which might be expected due to the variation inherent in MALDI analysis.

\section{Relative Ionization Yields}

Because of the large difference in signal between the tertiary and quaternary amines, a systematic study of the observed signal in a series of mixtures of PS with different end functionalities was conducted to determine their respective relative ionization efficiencies as a function of laser power and relative concentration. Total signal intensities for each component in the spectra from a mixture were obtained by integration of the respective major ion series (and the secondary series of the tertiary amine in the PSOH/PSA mixtures) and then summed separately without modification. The ratio was calculated from these values, and then multiplied by the inverse ratio of the number average molecular weights (from Table 1, with the quaternary form assumed to have same value as tertiary) to give the adjusted ratio to compare with the volume ratio.

In a previous study involving mixtures of polymers following MALDI analysis, Wood and coworkers describe an alternate approach, the use of monomer mole ratios to calculate the observed ratios [27]. In their approach, the total signal intensity is the sum of each observed oligomer intensity multiplied by the number of moles in that oligomer. Though this simplifies the comparison of mixtures of the same $\mathrm{w} / \mathrm{v}$ measurement between polymers of different molecular weight, the samples examined here are at similar, lower molecular weights, and have different end groups. The mass of the end groups can be a significant contributor to molar mass of the polymer at smaller molecular weights. For example, in our case, the end groups of the quaternary amine PS (butyl and trimethyl amino propyl) have a nominal mass of $158 \mathrm{u}$, nearly $10 \%$ of the number average molecular weight. Although our approach requires the assumption of correct molecular weight information, it also eliminates any error caused by the mass of the end groups, and simplifies calculations following integration.

The effect of laser power on relative ionization efficiency was first explored in order to determine whether there was any change over the power ranges normally used. MALDI spectral quality is highly dependent on the laser power. Without sufficient power, no peaks will be observed, and with too high power, fragmentation and skewing of the spectra can result [27]. If any skewing and/or increased fragmentation do 


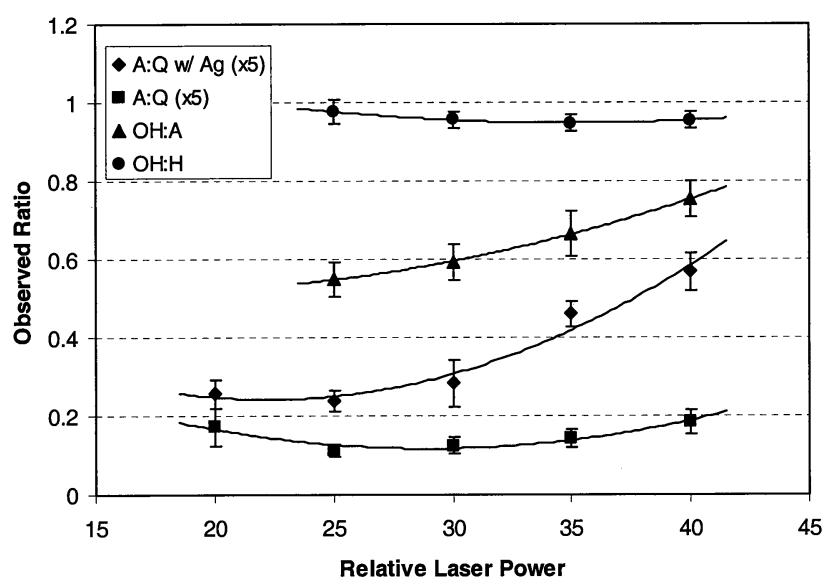

Figure 5. Signal intensity ratio of one to one mixtures of end functionalized PS as a function of laser power. Error bars represent one SD.

not equally effect both polymers in a mixture, the values for the relative ionization efficiency could change significantly with laser power. Figure 5 shows the experimentally determined relationship of the observed ratio of components in a mixture to the laser power. Each data point represents the average of the ratios obtained from nine spectra each of 1:1 mixtures of the end functionalized PS as indicated; because of the large difference in signal from the quaternary and tertiary amine samples, those results are multiplied by five for comparison.

The observed ratio of the PSOH to the PSH in Figure 5 is near unity, with other ratios being somewhat less. Both PSOH to PSH and PSA to PSQ without AgTFA show little variation as laser power increases. PSA to PSQ with AgTFA and PSOH to PSA, however, increase significantly with laser power. This may be due to the effect of metastable decay in the spectra of the tertiary amine sample when mixed with AgTFA. When in the same spectra with the quaternary sample, the metastable peaks are isobaric interferences with the $\mathrm{M}^{+}$peaks of the PSQ. Although one might expect the increasing metastable peak intensity as laser power increases to increase the apparent intensity of the PSQ signal, the opposite occurs. This may be because either the interference makes the PSQ signal more difficult to quantitate accurately (through peak broadening and subsequent baseline subtraction losses), or the signal attributable to the metastable fragment is too small at lower powers to have a measurable effect. In the case of the mixture of PSOH to PSA, the increasing fragmentation shown by the PSA decreases the relative signal as the power increases, causing the ratio to rise.

Although one can calculate the relative ionization efficiencies using the data in Figure 5, two issues make direct assignment of a value problematic. First, for the mixtures containing PSA with silver, the observed ratio varies dramatically with laser power. We make the assumption that the threshold laser power gives the most accurate representation of the mixture, and use

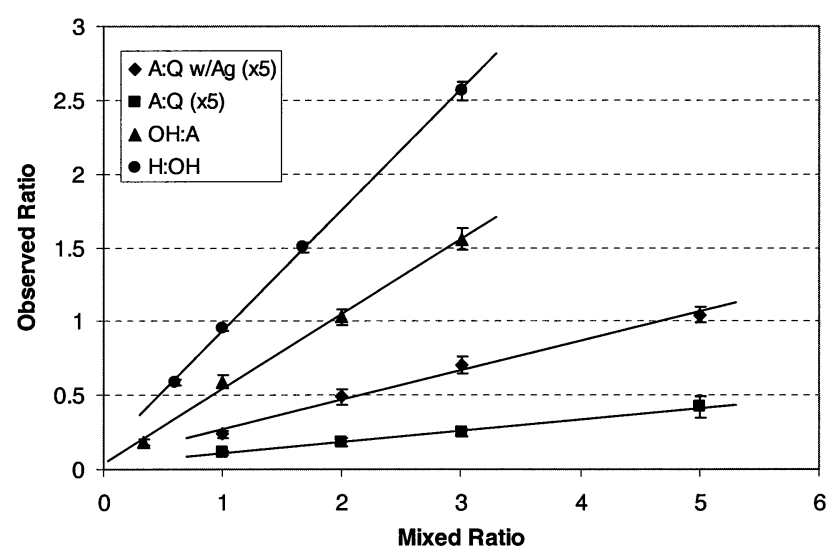

Figure 6. Signal intensity ratio of end functionalized PS mixtures as a function of mixture component ratio. Error bars represent one $\mathrm{SD}$.

spectra obtained at that power. Also, because the threshold laser power is usually selected for routine MALDI analysis, values at that power are most relevant. Secondly, because of the large difference in ionization efficiency between the quaternary amine PS and all the other functionalities, accurate quantitation of the other component in mixtures of PSQ is difficult, as the signal intensity is too low. For example, the signal from the tertiary amine is roughly one twentieth of that from the quaternary amine in a 1:1 mixture. Thus, mixtures with a lesser relative amount of PSQ must be used in order to provide comparable observed signal intensities and facile quantitation.

Figure 6 shows the calculated observed ratio for a number of different volume (or weight) ratios of the two components in each mixture. Each data point represents the average of nine spectra, all at a single laser power (from Figure 5, at 25), with the error bars representing one standard deviation from the mean. The plots are linear with an $\mathrm{R}^{2}$ value of 0.99 or greater. Although the range of concentrations used is limited because of lack of dynamic range in MALDI and the difficulty of integrating low intensity peaks, the trends are surprisingly linear. Differences in absolute intensity from spectrum to spectrum cause little variation in the relative intensities of the components. The reproducibility and linearity in the plots provides ample evidence that end group quantitation can be performed if other parameters (i.e., laser power, number of shots) are carefully considered.

The slopes of the linear regressions in Figure 6 represent the relative ionization efficiencies of the components in each mixture. By assuming that the ionization efficiency of the PSQ is $100 \%$, and comparing mixtures with a common component, the ionization efficiencies relative to PSQ of all the compounds can be determined, as shown in Table 3. For the tertiary amine, the ionization efficiency is $20 \%$ with AgTFA present, and $7.7 \%$ without. Both the hydroxyl and hydrogen terminated PS have nearly the same efficiency, 10 and 
Table 3. Ionization efficiencies relative to PSQ

\begin{tabular}{lcccc}
\hline PSH & PSOH & PSA & PSA w/ Ag & PSO \\
\hline \hline 0.095 & 0.10 & 0.077 & 0.20 & 1 \\
\hline
\end{tabular}

$9.5 \%$, respectively. Conversely, the relative ionization of PSQ is ten times that of PSH.

These results suggest that the ionization efficiency of a PS oligomer is only affected by the end group functionality if that functionality itself can be ionized. As cationization in a PS oligomer likely occurs in the middle of the monomer chain [9], this is not surprising. The identity of the end group would be expected to have little impact on the attachment of cation several monomers away. However, in the case where the end group can be charged under MALDI conditions, the impact on ionization efficiency is large. Thus, a convenient test to determine whether the ionization efficiencies are effected by the end group may be the presence of ionic species of different forms in a mixture, such as when a covalent cationized ion is observed rather than a metal cationized ion. Regardless, to assure proper quantitation, equal ionization efficiencies should not be assumed unless first tested with standard mixtures.

\section{Molecular Weight Differences}

Additional studies were conducted to examine the effect of molecular weight on ionization efficiency. As noted earlier, the silver cationization efficiency of PSA was found to increase with oligomer length. This could be problematic for quantification of polymer blends of diverse average molecular weight, or more polydisperse samples. Quantification of blends has been explored previously by Yan and coworkers, who found no molecular weight discrimination in ionization efficiencies of polydimethylsiloxane in the range of 1000 to $10,000 \mathrm{u}$ [27]. However, in that case, there was no difference in end functionality between blend components, and the oligomers ionize solely by protonation. Silver cationization of PS may be more susceptible to changes in oligomer length, and little is known about the behavior of PS which ionizes by other modes, such as covalent cationization.

With this in mind, the effects of the molecular weight and end functionality were explored with regard to each other. First, the effect of average molecular weight on the ionization efficiency was examined by comparing the observed ratios of standard mixtures of PSH, PSA, and PSQ at both 1600 and 2500 average molecular weights, as shown in Table 4. PSH was mixed with PSA at a one to one volume ratio, while PSA was mixed with PSQ both with and without AgTFA at a three to one ratio, in order to enhance the relative signal of the PSA for better quantitation. Little change is evident for the observed ratios of PSA to PSQ at the different molecular weights. PSA to PSQ with AgTFA has the same observed ratio at 1600 and 2500 average molecular
Table 4. Relative signal intensities of polymer mixture components

\begin{tabular}{lccc}
\hline $\begin{array}{l}\text { Sample } \\
\text { weight }\end{array}$ & PSH to PSA $^{\mathrm{a}}$ & PSA to PSQ $^{\mathrm{b}}$ & $\begin{array}{c}\text { PSA to PSQ w/ } \\
\text { AgTFA }^{\mathrm{b}}\end{array}$ \\
\hline \hline 1600 & $0.51 \pm 0.04$ & $0.049 \pm 0.005$ & $0.14 \pm 0.01$ \\
2500 & $0.92 \pm 0.04$ & $0.065 \pm 0.015$ & $0.14 \pm 0.01$ \\
\hline
\end{tabular}

a $1: 1 \mathrm{vol} / \mathrm{vol}$ ratio

b3:1 vol/vol ratio

weights, 0.14, while without AgTFA, the values differ slightly, 0.49 and 0.65 , respectively. The observed ratios of PSH to PSA differ dramatically depending on the molecular weight, increasing from 0.51 at 1600 to 0.92 at 2500.

The lack of change in the PSA to PSQ samples with AgTFA stands in contrast to the change in ionization efficiency shown in Figure 3 as oligomer length increases. This may be due to the overlap of the metastable ion series peaks of the PSA with the PSQ ion series peaks. A change in molecular weight may result in additional metastable ions, and a concurrent rise in the intensity of the PSQ peaks, eliminating any quantitative effect of the molecular weight change. The increase in PSH signal relative to PSA, though, may be explained by the change in ionization efficiency with oligomer length. If the silver cationization efficiency rises as molecular weight increases, while the contribution from protonation/depronation remains the same, the relative difference in ionization efficiency caused by the additional protonated/deprotonated species will decrease with increasing molecular weight. Regardless of the reason for the changes in ionization efficiency, the apparent dependence on molecular weight in some samples should give caution to those quantifying polymers of different average molecular weight.

Finally, the effect of the functional group on measured molecular weight itself was also examined. Because samples of amine end-functionalized PS can be ionized in any one of three modes, protonation/deprotonation, cationization, and covalently, from samples of nearly the same molecular weight, they provide a convenient system to use in exploration of any possible effects. Table 5 shows the number average molecular weights as determined by MALDI of PSA1600, PSA2500, and PSA 14200 in three different preparations: by itself, with silver, and after reaction to form the quaternary ammonium salt. Due to the overlap in the

Table 5. Number average molecular weights of tertiary and quaternary amine functionalized PS determined by MALDI

\begin{tabular}{|c|c|c|c|}
\hline \multirow{2}{*}{$\begin{array}{l}\text { Sample } \\
\text { Weight }\end{array}$} & \multicolumn{2}{|c|}{ Tertiary } & \multirow{2}{*}{$\begin{array}{l}\text { Quaternary } \\
\text { (w/o Ag) }\end{array}$} \\
\hline & w/o Ag & $\mathrm{w} / \mathrm{Ag}^{\mathrm{a}}$ & \\
\hline 1600 & $1665 \pm 5$ & $1717 \pm 3$ & $1676 \pm 9$ \\
\hline 2500 & $2520 \pm 8$ & $2631 \pm 18$ & $2595 \pm 12$ \\
\hline 14200 & $14253 \pm 36$ & $14597 \pm 33$ & $14405 \pm 57$ \\
\hline
\end{tabular}

${ }^{\mathrm{a} C o n t r i b u t i o n ~ f r o m ~} \mathrm{Ag}$ not subtracted. 
isotopic distributions of the protonated and silver cationized amine samples, the mass contribution from the silver cation was not subtracted out. Generally, the values for samples analyzed with AgTFA have the highest molecular weights among the groups, even given the mass contribution of the silver cation, followed by the quaternary samples, with the tertiary amine without AgTFA giving the lowest values. However, in light of a recent interlaboratory comparison in which the standard deviation observed for the number average weight of hydrogen terminated polystyrene in was $2 \%$ [17], the values in Table 5 are not significantly different. The end group functionality appears to have little difference on average molecular weight determination in this range.

\section{Conclusion}

The end group functionality of a polystyrene oligomer can have a significant impact on the matrix-assisted laser desorption-ionization properties of the polymer. Rather than having equal ionization efficiencies, polystyrenes terminated with functionalities that ionize, such as a "covalent cation", exhibit higher ionization efficiencies than polystyrenes with end groups that do not ionize, such as hydrogen, by as much as an order of magnitude. For the end groups studied here that do not confer additional modes of ionization, the ionization efficiencies are similar. The molecular weight of the polymer can also have significant impact on the ionization efficiency. The ionization efficiency of polystyrene oligomers was observed to change from oligomer to oligomer at masses below $3000 \mathrm{u}$, and was different in total between polystyrene samples of different molecular weight. However, little difference in average molecular weight was found between similar polystyrenes with different end-functionalities. Finally, when performing quantification of end groups in mixtures of polystyrenes, it should not be assumed that ionization efficiencies are the same. Quantification is possible, though, if proper care is exercised with regard to experimental conditions and the differing ionization efficiencies.

\section{Acknowledgments}

This research was supported by NSF grant CHE-9634238. Frederick Cox acknowledges support from an ACS Division of Analytical Chemistry Summer Graduate Fellowship. Arnab Dasgupta acknowledges support from the ACS Petroleum Fund, NSF grant 9973740, and the U.S. Army through the Center for Composite Materials at the University of Delaware. The authors thank Burnaby Munson and Michael Lassman for valuable discussion, and Sierra Analytics for the donation of software.

\section{References}

1. Rader, H. J.; Schrepp, W. MALDI-TOF Mass Spectrometry in the Analysis of Synthetic Polymers. Acta Polym. 1998, 49(6), 272-293.
2. Nielen, M. W. F. Maldi Time-of-Flight Mass Spectrometry of Synthetic Polymers. Mass Spectrom. Rev. 1999, 18, 309-344.

3. Hanton, S. D. Mass Spectrometry of Polymers and Polymer Surfaces. Chem. Rev. 2001, 101, 527-569.

4. Macha, S. F.; Limbach, P. A.; Hanton, S. D.; Owens, K. G. Silver Cluster Intereferences in Matrix-Assisted Laser Desorption/ Ionization (MALDI) Mass Spectrometry of Nonpolar Polymers. J. Am. Soc. Mass Spectrom. 2001, 12, 732-734.

5. Bauer, B. J.; Byrd, H. C. M.; Guttman, C. M. Small Angle Neutron Scattering Measurements of Synthetic Polymer Dispersions in Matrix-Assisted Laser Desorption/Ionization Matrixes. Rapid Commun. Mass Spectrom. 2002, 16, 1494-1500.

6. Trimpin, S.; Rouhanipour, A.; Az, R.; Rader, H. J.; Mullen, K. New Aspects in Matrix-Assisted Laser Desorption/Ionization Time-of-Flight Mass Spectrometry: A Universal Solvent-Free Sample Preparation. Rapid Commun. Mass Spectrom. 2001, 15, 1364-1373.

7. Deery, M. J.; Jennings, K. R.; Jasieczek, C. B.; Haddleton, D. M.; Jackson, A. T.; Yates, H. T.; Scrivens, J. H. A Study of Cation Attachment to Polystyrene by Means of Matrix-Assisted Laser Desorption/Ionization and Electrospray Ionization-Mass Spectrometry. Rapid Commun. Mass Spectrom. 1997, 11, 57-62.

8. Rashidezadeh, H.; Guo, B. Investigation of Metal Attachment to Polystyrenes in Matrix-Assisted Laser Desorption Ionization. J. Am. Soc. Mass Spectrom. 1998, 9, 724-730.

9. Gidden, J.; Bowers, M. T.; Jackson, A. T.; Scrivens, J. H. Gas-Phase Conformations of Cationized Poly(styrene) Oligomers. J. Am. Soc. Mass Spectrom. 2002, 13, 499-505.

10. Goldschmidt, R. J.; Wetzel, S. J.; Blair, W. R.; Guttman, C. M. Post-Source Decay in the Analysis of Polystyrene by MatrixAssisted Laser Desorption/Ionization Time-of-Flight Mass Spectrometry. J. Am. Soc. Mass Spectrom. 2000, 11, 1095-1106.

11. Scrivens, J. H.; Jackson, A. T.; Yates, H. T.; Green, M. R.; Critchley, G.; Brown, J.; Bateman, R. H.; Bowers, M. T.; Gidden, J. The Effect of the Variation of Cation in the MatrixAssisted Laser Desorption/Ionization-Collision Induced Dissociation (MALDI-CID) Spectra of Oligomeric Systems. Int. J. Mass Spectrom. Ion Processes 1997, 165/166, 363-375.

12. Jackson, A. T.; Yates, H. T.; Scrivens, J. H.; Green, M. R.; Bateman, R. H. Matrix-Assisted Laser Desorption/IonizationCollision Induced Dissociation of Poly(styrene). J. Am. Soc. Mass Spectrom. 1998, 9, 269-274.

13. Jackson, A. T.; Bunn, A.; Hutchings, L.R.; Kiff, F. T.; Richards, R. W.; Williams, J.; Green, M. R.; Bateman, R. H. The Generation of End Group Information from Poly(styrene)s by Means of Matrix-Assisted Laser Desorption/Ionization-Collision Induced Dissociation. Polymer 2000, 41, 7437-7450.

14. Shimada, K.; Lusenkova, M. A.; Sato, K.; Saito, T.; Matsuyama, S.; Nakahara, H.; Kinugasa, S. Evaluation of Mass Discrimination Effects in the Quantitative Analysis of Polydisperse Polymers by Matrix-Assisted Laser Desorption/Ionization Time-of-Flight Mass Spectrometry Using Uniform Oligomers. Rapid Commun. Mass Spectrom. 2001, 15, 277-282.

15. Schriemer, D. C.; Li, L. Detection of High Molecular Weight Narrow Polydisperse Polymers Up to 1.5 Million Daltons by MALDI Mass Spectrometry. Anal. Chem. 1996, 68, 2721-2725.

16. Zhu, H.; Yalcin, T.; Li, L. Analysis of the Accuracy of Determining Average Molecular Weights of Narrow Polydispersity Polymers by Matrix-Assisted Laser Desorption Ionization Time-of-Flight Mass Spectrometry. J. Am. Soc. Mass Spectrom. 1998, 9, 275-281.

17. Guttman, C. M.; Wetzel, S. J.; Blair, W. R.; Fanconi, B. M.; Girard, J. E.; Goldschmidt, R. J.; Wallace, W. E.; Vanderhart, D. L. NIST-Sponsored Interlaboratory Comparison of Polystyrene Molecular Mass Distribution Obtained by MatrixAssisted Laser Desorption/Ionization Time-of-Flight Mass 
Spectrometry: Statistical Analysis. Anal. Chem. 2001, 73, 12521262.

18. Quirk, R. P.; Mathers, R. T.; Wesdemiotis, C.; Arnould, M. A. Investigation of Ethylene Oxide Oligomerization during Functionalization of Poly(styryl)lithium Using MALDI-TOF MS and NMR. Macromolecules 2002, 35, 2912-2918.

19. Zammit, M. D.; Davis, T. P.; Haddleton, D. M.; Suddaby, K. G. Evaluation of the Mode of Termination for a Thermally Initiated Free-Radical Polymerization Via Matrix-Assisted Laser Desorption Ionization Time-of-Flight Mass Spectrometry. Macromolecules 1997, 30, 1915-1920.

20. Belu, A. M.; DeSimone, J. M.; Linton, R. W.; Lange, G. W.; Friedman, R. M. Evaluation of Matrix-Assisted Laser Desorption Ionization Mass Spectrometry for Polymer Characterization. J. Am. Soc. Mass Spectrom. 1996, 7, 11-24.

21. Weaver, A. P.; Guttman, C. M.; Girard, J. E. Quantitation of Polymer End Groups Using MALDI TOF MS. Presented at the 50th ASMS Conference, Orlando, FL, June 2002, Abstract No. 1054.

22. Alicata, R.; Montaudo, G.; Puglisi, C.; Samperi, F. Influence of Chain End Groups on the Matrix-Assisted Laser Desorption/ Ionization Spectra of Polymer Blends. Rapid Commun. Mass Spectrom. 2002, 16, 248-260.

23. Puglisi, C.; Samperi, F.; Alicata, R.; Montaudo, G. EndGroups-Dependent MALDI Spectra of Polymer Mixtures. Macromolecules 2002, 35, 3000-3007.

24. Bauer, B. J.; Wallace, W. E.; Fanconi, B. M.; Guttman, C. M. Covalent Cationization Method for the Analysis of Polyethylene by Mass Spectrometry. Polymer 2001, 42, 9949-9953.

25. Lin-Gibson, S.; Brunner, L.; Vanderhart, D. L.; Bauer, B. J.; Fanconi, B. M.; Guttman, C. M.; Wallace, W. E. Optimizing the
Covalent Cationization Method for the Mass Spectrometry of Polyolefins. Macromolecules 2002, 35, 7149-7156.

26. Ji, H.; Sato, N.; Nakamura, Y.; Wan, Y.; Howell, A.; Thomas, Q. A.; Storey, R. F.; Nonidez, W. K.; Mays, J. W. Characterization of Polyisobutylene by Matrix-Assisted Laser Desorption Ionization Time-of-Flight Mass Spectrometry. Macromolecules 2002, 35, 1196-1199.

27. Yan, W.; Gardella, J. A.; Wood, T. D. Quantitative Analysis of Technical Polymer Mixtures by Matrix Assisted Laser Desorption/Ionization Time of Flight Mass Spectrometry. J. Am. Soc. Mass Spectrom. 2002, 13, 914-920.

28. Dasgupta, A.; Kurian, M.; Galvin, M.; Beyer, F. L. unpublished.

29. Quirk, R. P.; Lee, Y. Quantitative Amine Functionalization of Polymeric Organolithium Compounds with 3-Dimethylaminopropyl Chloride in the Presence of Lithium Chloride. J. Polym. Sci. A 2000, 38, 145.

30. Cox, F. J.; Johnston, M. V.; Qian, K.; Peiffer, D. G. Proceedings of the 48th ASMS Conference, Dallas, TX, June 2000, Abstract No. 1251

31. Ji, H.; Sato, N.; Nonidez, W. K.; Mays, J. W. Characterization of Hydroxyl-End-Capped Polybutadiene and Polystyrene Produced by Anionic Polymerization Technique via TLC/MALDI TOF Mass Spectrometry. Polymer 2002, 43, 7119-7123.

32. Lias, S. G.; Levin, R. D.; Liebman, J. F. Ion Energetics Data; NIST Chemistry WebBook: NIST Standard Reference Database Number 69. Linstrom, P. J.; Mallard, W. G. Eds.; National Institute of Standards and Technology, Gaithersburg, July 2001; http:/ / webbook.nist.gov. 